typeset using JPSJ.sty $<$ ver.1.0b $>$

\title{
Induced-Moment Weak Antiferromagnetism and Orbital Order on the Itinerant-Localized Duality Model with Nested Fermi Surface: A Possible Origin of Exotic Magnetism in $\mathrm{URu}_{2} \mathrm{Si}_{2}$
}

\author{
Yukihiro OKUnO ${ }^{1,2}$ G and Kazumasa MrYAKE ${ }^{2}$ \\ ${ }^{1}$ Department of Physics, Kyoto University, Kyoto 606-01 \\ ${ }^{2}$ Department of Physical Science, Graduate School of Engineering Science, Osaka University, Toyonaka 560
}

(Received )

\begin{abstract}
The weak antiferromagnetism of $\mathrm{URu}_{2} \mathrm{Si}_{2}$ is discussed on the basis of a duality model which takes into account salient features of both itinerant fermions and "localized" component of spin degrees of freedom. The problem is analyzed in the framework of induced-moment mechanism by taking a singlet-singlet crystal field scheme together with the nesting property of partial Fermi surface of itinerant fermions. It is shown that the extremely small ordered moment $m$ of $\mathcal{O}\left(10^{-2} \times \mu_{\mathrm{B}}\right)$ can be compatible with the large specific-heat jump at the transition temperature $T_{\mathrm{N}}$. Analysis performed in the presence of external magnetic field shows that the field dependence of $m$ in the limit $T \rightarrow 0$ and $T_{\mathrm{N}}$ do not scale except very near the critical field $B_{\mathrm{c}}$ which is consistent with a recent observation by Mentink et al. It is also shown that the antiferromagnetic magnetic order gives rise to a tiny amount of antiferromagnetic orbital order of f-electrons.
\end{abstract}

KEYWORDS: induced-moment magnetism with nesting, weak antiferromagnetism of $\mathrm{URu}_{2} \mathrm{Si}_{2}$, itinerant-localized duality, orbital order

\section{§1. Introduction}

One of the major problems which have not yet been resolved in heavy Fermions is how to understand the exotic nature of extremely weak magnetism of $\mathrm{URu}_{2} \mathrm{Si}_{2}$ which also exhibits a superconducting transition at the lower temperature 1 The most fundamental question is why the ordered moment $m$ is so small of the order of $0.04 \mu_{\mathrm{B}}$, 陑 while the specific heat exhibits a large jump of the order of that in the normal state at the transition temperature which we call $T_{\mathrm{N}}$. $⿴$. $\mathrm{E}$ Moreover, it has recently been reported that the magnetic-filed dependence of $T_{\mathrm{N}}$ and $m$ in the limit $T \rightarrow 0$ do not seem to scale with each other but seem to have different critical fields.6) At a glance these facts appear to indicate that the true order parameter of this transition is not magnetic but some hidden one which has an intimate relation with the degrees of charge polarization $\mathbb{W}$

\footnotetext{
* Present adress: Yukawa Institute for Theoretical Physics, e-mail: okuno@yukawa.kyoto-u.ac.jp
} 
However, before we look for such novel mechanisms, it might still make sense to investigate to what extent these anomalies can be understood by extending the conventional treatment for magnetic mechanism so as to take into account the specific nature of $\mathrm{URu}_{2} \mathrm{Si}_{2}$. In $\mathrm{URu}_{2} \mathrm{Si}_{2}$ the ionic state of $\mathrm{U}^{+4}$ with $5 \mathrm{f}^{2}$ is considered to be realized. Then the state with total angular momentum $J=4$, which is formed by the Hund-rule coupling and strong spin-orbit interaction, is split by the crystal field effect lifting the 9-fold degeneracy into five singlets and two doublets in general. It does not seem unreasonable to assume that the ground state consists of singlet which has large matrix element of angular momentum between the excited singlet state. For instance, the singlet-singlet model was adopted to explain the temperature dependence of the bulk magnetic susceptibility and the heat capacity, (⿴囗⿱一一) ) and the collective excitations measured by the inelastic neutron scattering. (10)

Although the singlet ground state has no average magnetic moment, the so-called inducedmoment mechanism can work to cause the ordered state by invoking the virtual process of mixing between the ground state and the low-lying excited states of crystal field. The ordered moment so obtained is rather reduced below its full moment in general 11, 12) However, the usual inducedmoment mechanism on the localized model is not enough to understand simultaneously the extremely small magnetic moment of the order of $1 \%$ of the full uranium moment and the large specific heat jump at $T_{\mathrm{N}}$.

In this paper we demonstrate that the fundamental property of "magnetic transition" of $\mathrm{URu}_{2} \mathrm{Si}_{2}$ can be understood by the induced-moment mechanism on the basis of a itinerant-localized duality model together with an assumption of the nesting of the part of Fermi surface. Sikkema et al have already proposed the model with the nested Fermi surface on the singlet-singlet crystal field scheme for the weak-moment formation of $\mathrm{URu}_{2} \mathrm{Si}_{2}{ }_{13}$ Their model, an Ising-Kondo lattice model with transverse field which originates from the off-diagonal element of two singlet levels, produces weak moment but rather small transition temperature and does not reproduce the large specific heat jump. Our scheme here shows that the tiny moment of antiferomagnetic order does not contradict with large specific heat jump at $T=T_{\mathrm{N}}$, and the unusual magnetic-field dependence of $m(T \rightarrow 0)$ and $T_{\mathrm{N}}$ can be reproduced within the order of magnitude for a reasonable set of parameters. It is also shown that the charge distributions of f-electrons at two sublattice sites in the antiferromagnetic state are different giving rise to a tiny amount of antiferromagnetic orbital order.

\section{$\S 2 . \quad$ Formalism}

\subsection{Outline of itinerant-localized duality model}

An itinerant-localized duality model has been proposed by Kuramoto and Miyake 14, (55) as a quantum phenomenology in order to explain the properties of heavy Fermions beyond the conventional Fermi liquid such as the weak antiferromagnetism and metamagnetism. Quite recently, an investi-

gation to examine its microscopic basis was put forth 16) The problem about exotic magnetism of 
$\mathrm{URu}_{2} \mathrm{Si}_{2}$ seems to within the scope which the duality model can be applied as was already argued in its simple fasion. 15 )

In the "duality model", the partition function $\mathrm{Z}$ and the effective action $\mathrm{A}$ is given as 14,15 )

$$
\begin{aligned}
Z & =\int \mathrm{D} f^{\dagger} \int \mathrm{D} f \int \mathrm{D} \operatorname{Sexp}(-\beta A) \\
A & =A_{\mathrm{f}}+A_{\mathrm{s}}+A_{\mathrm{int}},
\end{aligned}
$$

where $A$ 's are defined in terms of $f$ and $f^{\dagger}$, Grassmann numbers, and $\mathbf{S}, c$-number vector, as follows:

$$
\begin{aligned}
A_{\mathrm{f}} & =-\sum_{i, j . \sigma} \sum_{n} f_{i \sigma}^{\dagger}\left(-\mathrm{i} \epsilon_{n}\right)\left(G_{i j, \sigma}^{-1}\left(\mathrm{i} \epsilon_{n}\right)\right) f_{j \sigma}\left(\mathrm{i} \epsilon_{n}\right), \\
A_{\mathrm{s}} & =\frac{1}{2} \sum_{i, j, m} \mathbf{S}_{i}\left(-\mathrm{i} \nu_{m}\right)\left(\chi_{0 i j}^{-1}\left(i \nu_{m}\right) \delta_{i j}-J_{i j}\right) \mathbf{S}_{j}\left(\mathrm{i} \nu_{m}\right)-\sum_{i} h_{i} S_{i z} \\
A_{\text {int }} & =-\lambda_{0} \sum_{i \alpha \beta} \sum_{m n} f_{i \alpha}^{\dagger}\left(-\mathrm{i} \epsilon_{n}-\mathrm{i} \nu_{m}\right) f_{i \beta}\left(\mathrm{i} \epsilon_{n}\right) \vec{\sigma}_{\alpha \beta} \cdot \mathbf{S}_{i}\left(\mathrm{i} \nu_{m}\right),
\end{aligned}
$$

where $A_{\mathrm{f}}$ represents the part of itinerant fermions, $A_{\mathrm{s}}$ the localized component of spin degrees of freedom consisting of incoherent part of fermions, and $A_{\text {int }}$ is the interaction between the fermion and the "localized spin". $G\left(\mathrm{i} \epsilon_{n}\right)$ in $A_{\mathrm{f}}$, eq. $(2.2)$, is the Green function of the itinerant fermion and $\chi_{0}\left(\mathrm{i} \nu_{m}\right)$ in $A_{\mathrm{s}}$, eq.(2.2), is the partially renormalized local spin susceptibility which does not include the effect of neither the RKKY interaction and nor the coupling with fermions. The exchange interaction between "localized spins" is represented by $J_{i j}$, and a magnetic field at site $i$ is denoted by $h_{i}$. Although the above form of the action is isotropic in spin space we retain only one component, $S_{z}$, because the large uniaxial magnetic anisotropy exists in the case we discuss below. So in the discussion below we only retain one component of spin, $S_{z}$.

In order to discuss the magnetic properties of the strongly correlated systems, we first take the trace over $f^{\dagger}$ and $f$ as follows:

$$
\begin{aligned}
Z & =\operatorname{det} G \times \int \mathrm{D} S \exp \left(-\beta A_{\mathrm{m}}\right) \\
A_{\mathrm{m}} & =A_{\mathrm{s}}-\frac{1}{\beta} \operatorname{Tr} \ln \left(1+\lambda_{0} G \sigma_{z} \cdot S_{z}\right) .
\end{aligned}
$$

As a first step of approximation, we take the saddle-point approximation for the macroscopic mode $\mathbf{S}_{q}$ (with the wavevector $q=0$ or the antifferomaginetic wavevector $Q$ ) and the Gaussian average with respect to other modes of spin fluctuations. Then, we obtain the equations of states in the form

$$
\begin{aligned}
{\left[\frac{1}{\chi_{0}(0, S)}-J(0)-2 \lambda_{0}^{2} \Pi\left(0, S_{0}\right)\right] S_{0} } & =h \\
\frac{1}{\chi_{0}(Q, S)}-J(Q)-2 \lambda_{0}^{2} \Pi\left(Q, S_{Q}\right) & =0
\end{aligned}
$$


where $J(q)$ (with $q=0$ or $Q$ ) is the Fourier component of the exchange interaction $J_{i j}$, and $\chi_{0}(q, \mathbf{S}$ ) is the Fourier component of the static "local susceptibility" which has site dependence in general. When we take into account only the nearest-neighbor interaction, $J(0)=-z J$ and $J(Q)=z J$, $z$ being the number of nearest neighbors. It is noted that the local susceptibility $\chi_{0 i i}$ has a site dependence due to the mean field of antiferromagnetic induced moment, in general, as discussed below. As a result, $\chi_{0}$ has a wavenumber dependence as in eqs.(2.4). The polarization function $\Pi\left(q, \mathbf{S}_{q}\right)$ of the itinerant component is given as

$$
\Pi\left(q, \mathbf{S}_{q}\right)=-\frac{1}{N} \sum_{\mathbf{k}} \sum_{\epsilon_{n}}\left[G_{\sigma}\left(\mathbf{k}, \mathrm{i} \epsilon_{n}\right)^{-1} G_{\sigma}\left(\mathbf{k}+\mathbf{q}, \mathrm{i} \epsilon_{n}\right)^{-1}-\left(\lambda \mathbf{S}_{q}\right)^{2}\right]^{-1} .
$$

\subsection{Induced-moment antiferromagnetism}

A structure of eq.(2.4b) is the same as the mean field equation in the induced-moment mechanism except for the effective exchange interaction due to the polarization $\Pi\left(q, S_{Q}\right)$. In the latter mechanism, $\chi_{0}$ is given by the so-called Van Vleck susceptibility arising from the virtual excitation between the singlet ground state and some excited state of crystal field levels.11, 12) In the present problem, $\chi_{0}$ is considered to consist both of such local Van Vleck susceptibility and Kondo like correlation arising from the effect of hybridization between the localized f-electron and the conduction electrons in general. In order to obtain the extremely small ordered moment $m \sim \mathcal{O}\left(10^{-2} \times \mu_{\mathrm{B}}\right)$, the conventional induced-moment mechanism is not enough unless the exchange interaction $J(Q)$ almost coincides accidentally with its threshold for the occurrence of ordered state.12 Furthermore, only a tiny jump in the specific heat can be expected in such a situation. So, the effect of polarization should be crucial as pointed out in ref.15. We evaluate $\Pi\left(q, \mathbf{S}_{q}\right)$, eq.(2.5), with use of the quasi-particle form for $G_{\sigma}\left(k, \mathrm{i} \epsilon_{n}\right)$ :

$$
G_{\sigma}\left(k, \mathrm{i} \epsilon_{n}\right)=\frac{a_{\mathrm{f}}}{\mathrm{i} \epsilon_{n}-\sigma \tilde{h}-E_{k}},
$$

where $a_{\mathrm{f}}$ is the renormalization factor, $E_{k}$ is the energy spectrum of the itinerant fermion measured from the chemical potential, and $\tilde{h}=a_{\mathrm{f}} h$ is the renormalized external field. It is noted that the renormalized field $\tilde{h}$ is very small value $(\tilde{h}<<h)$ in the strongly correlated regime, while the "localized spin" is affected directly by the external field $h$ which affects the itinerant fermion through the coupling $\lambda_{0}$ in, $A_{\text {int }}$, eq.(2.2). As for the form of $\chi_{0}$, the local spin susceptibility, we take so as to reproduce the Van Vleck susceptibility on the singlet-singlet scheme neglecting the effect of Kondo like correlation. As the suitable form of the local susceptibility, $\chi_{0}(0, S)$ and $\chi_{0}(Q, S)$, we take those form in the case of induced moment as explained below.

Assuming the two sublattices $A$ and $B$, the molecular-field equation of induced-moment antifer- 
romagnetism is given as follow:12)

$$
\begin{aligned}
S_{A} & =\frac{-2\left(z J S_{B}-h\right) \mathrm{c}^{2}}{\sqrt{\Delta^{2}+\left(2 z J S_{B}-2 h\right)^{2} \mathrm{c}^{2}}} \operatorname{th} \frac{\sqrt{\Delta^{2}+\left(2 z J S_{B}-2 h\right)^{2} \mathrm{c}^{2}}}{2 T} \\
S_{B} & =\frac{-2\left(z J S_{A}-h\right) \mathrm{c}^{2}}{\sqrt{\Delta^{2}+\left(2 z J S_{A}-2 h\right)^{2} \mathrm{c}^{2}}} \operatorname{th} \frac{\sqrt{\Delta^{2}+\left(2 z J S_{A}-2 h\right)^{2} \mathrm{c}^{2}}}{2 T},
\end{aligned}
$$

where $S_{A}$ and $S_{B}$ are the induced moment at the site $A$ and $B$, respectively. $\Delta$ denotes the energy difference between the ground-state singlet, $|0\rangle$, and the excited-state singlet, $|1\rangle$, and $\mathrm{c} \equiv\left\langle 1\left|\mathrm{~J}_{z}\right| 0\right\rangle$, which is an essential ingredient of the Van Vleck susceptibility. In deriving eqs.(2.7a) and (2.7b), we have noted that the effective field at the site $\mathrm{A}$ is given by $h_{A \text { eff }}=h-z J S_{B}$, and that at the site $\mathrm{B}$ is $h_{B \text { eff }}=h-z J S_{A}$, respectively.

The local spin susceptibility $\chi_{0}(0, S)$ 's in eqs. $(2.4 \mathrm{a})$ and $(2.4 \mathrm{~b})$ are defined from the relations as follows:

$$
\begin{aligned}
S_{0} & =h_{0} \chi_{0}(0, S) \\
S_{Q} & =h_{Q} \chi_{0}(Q, S),
\end{aligned}
$$

where $h_{q}$ and $S_{q}$, with $q=0$ or $Q$, are the Fourier component of the effective field and the induced moment, respectively, defined as

$$
\begin{aligned}
h_{0} & =\frac{1}{N} \sum_{r_{i}} \mathrm{e}^{\mathrm{i} 0 \cdot r_{i}} h_{\mathrm{eff}, i}=\frac{1}{2}\left(h_{\mathrm{eff}, A}+h_{\mathrm{eff}, B}\right)=h-\frac{1}{2}\left(z J S_{A}+z J S_{B}\right) \\
h_{Q} & =\frac{1}{N} \sum_{r_{i}} \mathrm{e}^{\mathrm{i} Q \cdot r_{i}} h_{\mathrm{eff}, i}=\frac{1}{2}\left(h_{\mathrm{eff}, A}-h_{\mathrm{eff}, B}\right)=\frac{1}{2}\left(z J S_{A}-z J S_{B}\right) \\
S_{0} & =\frac{1}{N} \sum_{r_{i}} \mathrm{e}^{\mathrm{i} 0 \cdot r_{i}} S_{i}=\frac{1}{2}\left(S_{A}+S_{B}\right) \\
S_{Q} & =\frac{1}{N} \sum_{r_{i}} \mathrm{e}^{\mathrm{i} Q \cdot r_{i}} S_{i}=\frac{1}{2}\left(S_{A}-S_{B}\right) .
\end{aligned}
$$

Here susceptibility defined above contains the non-linear components of effective field in general. However, when the effective field is small as in the present case, we can approximate it as the linearized susceptibility. Then, substituting (2.9a) [(2.9b)] and (2.9c) [(2.9d)] with (2.7a) and (2.7b), we obtain the expression for the susceptibility $\chi_{0}(0, S)\left[\chi_{0}(Q, S)\right]$ as follows:

$$
\begin{aligned}
\chi_{0}(0, S) & =\frac{\frac{1}{2}\left(h-z J S_{B}\right) \chi_{A}+\frac{1}{2}\left(h-z J S_{A}\right) \chi_{B}}{h-\frac{1}{2}\left(z J S_{A}+z J S_{B}\right)} \\
\chi_{0}(Q, S) & =\frac{\frac{1}{2}\left(h-z J S_{B}\right) \chi_{A}-\frac{1}{2}\left(h-z J S_{A}\right) \chi_{B}}{\frac{1}{2}\left(z J S_{A}-z J S_{B}\right)}
\end{aligned}
$$

where $\chi_{A}$ and $\chi_{B}$ are defined as

$$
\begin{aligned}
\chi_{A} & =\frac{2 \mathrm{c}^{2}}{\sqrt{\Delta^{2}+\left(2 z J S_{B}-2 h\right)^{2} \mathrm{c}^{2}}} \operatorname{th} \frac{\sqrt{\Delta^{2}+\left(2 z J S_{B}-2 h\right)^{2} \mathrm{c}^{2}}}{2 T} \\
\chi_{B} & =\frac{2 \mathrm{c}^{2}}{\sqrt{\Delta^{2}+\left(2 z J S_{A}-2 h\right)^{2} \mathrm{c}^{2}}} \operatorname{th} \frac{\sqrt{\Delta^{2}+\left(2 z J S_{A}-2 h\right)^{2} \mathrm{c}^{2}}}{2 T} .
\end{aligned}
$$




\section{§3. Analysis of the Model}

First of all, let us assess the size of parameters appearing in the above formulae keeping it in mind to applying them to $\mathrm{URu}_{2} \mathrm{Si}_{2}$. The crystal field parameter $\Delta$ and the matrix element $c$ can be estimated so as to reproduce the temperature dependence of susceptibility in high temperature region. In Niewenhuys's scheme of crystal field $80=1.2 \times 10^{2} \mathrm{~K}$ and $c=1.2 \mu_{\mathrm{B}}$, the values of which have been frequently used by analysis. On the other hand, in the scheme of Santini et al's, , $\mathrm{G}$ ) $\Delta=4.6 \times 10^{2}$ $\mathrm{K}$ and $c=1.6 \mu_{\mathrm{B}}$, the values of which give better agreement with experiment. Therefore, we adopt the latter scheme by Santini et al. In the latter crystal field scheme, there exist doublet levels between the ground and the excited singlet levels, and this fact explains naturally the large entropy at low temperature leading to the large specific heat. The exchange interaction $J$ is chosen so as to fit the neutron scattering data on the basis of RPA theory of singlet-singlet model 10 ) The 'band' width of itinerant fermion, $2 D$, is of the order of $T_{\text {coh }}$ which is about $100 \mathrm{~K}$ for $\mathrm{URu}_{2} \mathrm{Si}_{2}$. The renormalized spin-fermion coupling $\tilde{\lambda}$ is the same order of $T_{\text {coh }}=T_{\mathrm{K}}$. 14) In the calculations below, we set the matrix element c and $D$ as unit of magnetization and of energy, respectively. Here we remark about the values of parameters. Since various assumptions are involved in the above estimation, there remains ambiguity about these values. In particular the matrix element $c$ is difficult to evaluate and actual value may be possibly smaller than the estimated one. So is the crystal field splitting $\Delta$, and it can be much larger. We discuss these points later.

\subsection{Ordered moment at zero temperature}

When the external field is absent, $h=0$, the uniform component of magnetic moment $S_{0}=0$. Then, by substituting (2.6) into (2.5), the polarization $\Pi$ in eq. $(2.4 \mathrm{~b})$ is expressed as

$$
\Pi\left(Q, S_{Q}\right)=-\frac{a_{\mathrm{f}}^{2}}{N} T \sum_{\epsilon_{n}} \sum_{\mathbf{k}}\left[\left(\mathrm{i} \epsilon_{n}-E_{\mathbf{k}}\right)\left(\mathrm{i} \epsilon_{n}-E_{\mathbf{k}+\mathbf{Q}}\right)-\left(\tilde{\lambda} S_{Q}\right)^{2}\right]^{-1},
$$

where $\tilde{\lambda} \equiv a_{\mathrm{f}} \lambda_{0}$. If the nesting condition $E_{\mathbf{k}+\mathbf{Q}}=-E_{\mathbf{k}}$ is fulfilled, (3.1) is easily evaluated as

$$
\Pi\left(Q, S_{Q}\right)=\frac{1}{2} a_{\mathrm{f}}^{2} \rho_{\mathrm{F}} \int_{-D}^{D} \frac{\mathrm{d} \epsilon}{\sqrt{\epsilon^{2}+\left(\tilde{\lambda} S_{Q}\right)^{2}}} \operatorname{th} \frac{\sqrt{\epsilon^{2}+\left(\tilde{\lambda} S_{Q}\right)^{2}}}{2 T},
$$

where $\rho_{\mathrm{F}}$ is the density of states at the Fermi level and $D$ is half the bandwidth of itinerant fermion.

Hereafter, we use $m$ for the staggered magnetization, i.e., $S_{Q}=m$. At $T=0, \Pi(0, m)$ is estimated as

$$
\Pi(Q, m)=a_{\mathrm{f}}^{2} \rho_{\mathrm{F}} \log \frac{2 D}{\tilde{\lambda} m} .
$$


The local susceptibility $\chi_{0}$ in eq.(2.4b) at $T=0$ is evaluated from $(2.11 \mathrm{~b})$ as

$$
\begin{aligned}
\chi_{0}\left(0, S_{Q}\right) & =\frac{2 \mathrm{c}^{2}}{\sqrt{\Delta^{2}+(2 z J \mathrm{~cm})^{2}}} \\
& \simeq \frac{2 \mathrm{c}^{2}}{\Delta} .
\end{aligned}
$$

The last approximation in (3.4) holds when the level splitting $\Delta$ of crystal field is far larger than $2 z J \mathrm{~cm}$.

Thus the equation of state $(2.4 \mathrm{~b})$ at $T=0$ is reduced to

$$
\frac{\sqrt{\Delta^{2}+(2 z J \mathrm{c} m)^{2}}}{2 \mathrm{c}^{2}}-z J-2 \tilde{\lambda}^{2} \rho_{\mathrm{F}} \log \frac{2 D}{\tilde{\lambda} m}=0 .
$$

If the approximation of $(3.4 \mathrm{~b})$ is valid, we can estimate the size of the magnetization $m$ at $T=0$ as

$$
m \simeq \frac{2 D}{\tilde{\lambda}} \exp \left[-\frac{1}{2 \tilde{\lambda}^{2} \rho_{\mathrm{F}}}\left(\frac{\Delta}{2 \mathrm{c}^{2}}-z J\right)\right],
$$

which is meaningful only in the case $\Delta>2 z J c^{2}$, where the ordered state is not realized without a help of the polarization $\Pi$ because the conventional condition for the induced-moment ordering to occur is given by $\Delta / 2 \mathrm{c}^{2}<z J$. The ordered moment given by (3.6) can become extremely small only for the small value of the local susceptibility, i.e., $\chi_{0}(Q, S) / \rho_{\mathrm{F}} \simeq 2 c^{2} / \Delta \rho_{\mathrm{F}} \ll 1$ because $\tilde{\lambda} \rho_{\mathrm{F}} \sim 1$ and $D / \tilde{\lambda} \sim 1$ as mentioned above. It is noted that such an extremely small ordered moment is hard to be realized only from the usual nesting property of itinerant fermions unless $T_{\mathrm{N}} \ll D$ which is not the case in the present problem. While the ordered moment is extremely small, the specific heat exhibits a rather large jump at $T=T_{\mathrm{N}}$ of the order of $C_{\mathrm{n}}\left(T_{\mathrm{N}}\right)$, the specific heat at the normal side, as will be discussed below. This is because the mathematical structure of the thermodynamic potential in the nesting system is similar to that of the superconductivity.

\subsection{Temperature dependence of ordered moment}

The temperature dependence of $m$ is obtained from the relation (2.4b)

$$
\frac{\sqrt{\Delta^{2}+(2 z J m \mathrm{c})^{2}}}{2 \mathrm{c}^{2}} \operatorname{cth} \frac{\sqrt{\Delta^{2}+(2 z J m \mathrm{c})^{2}}}{2 T}-z J-\tilde{\lambda}^{2} \rho_{\mathrm{F}} \int_{-D}^{D} \frac{\mathrm{d} \epsilon}{\sqrt{\epsilon^{2}+(\tilde{\lambda} m)^{2}}} \operatorname{th} \frac{\sqrt{\epsilon^{2}+(\tilde{\lambda} m)^{2}}}{2 T}=0
$$

where we have used $(3.2)$ for $\Pi(Q, m)$ and $(2.10 \mathrm{~b})$ for $\chi_{0}(0, m)$ with $(2.11)$ substituted by $h=0$. When the transition temperature is small compared to $\Delta$ we can neglect the $m$ dependence of the localized-spin part of susceptibility and can estimate the transition temperature as,

$$
T_{N} \simeq 2.26 D \exp \left[-\frac{1}{2 \tilde{\lambda}^{2} \rho_{\mathrm{F}}}\left(\frac{\Delta}{2 \mathrm{c}^{2}}-z J\right)\right] .
$$

So we can get the relation $T_{N} \simeq 1.13 \tilde{\lambda} m$ from (3.6) 15 Considering that the $\tilde{\lambda}$ is the order of $T_{\text {coh }}$, we expect relatively large value of transition temperature compare to the magnitude of the moment 
from this scaling relation.

Numerical solutions of (3.7) are shown in Fig. 1 where the temperature dependence of the magnetization $m$ for various values of $\Delta$ and $\tilde{\lambda}$ are drawn. The realization of small value of magnetization stands in the delicate balance of various parameters which is considered to be realized in $\mathrm{URu}_{2} \mathrm{Si}_{2}$. One peculiar feature of $\mathrm{URuSi}_{2}$ is the extremely small value of magnetization compared to rather 'high' transition temperature $T_{N}$. In order to obtain the extremely small magnetization $m$ but not so small value of $T_{N}$, the equations (3.6) and (3.8) suggest that it requires not only large value of spin-fermion coupling $\tilde{\lambda}$ but also the large value of crystal field splitting. Recalling that we have taken $c$ and $D$ as the unit of the value of magnetization and energy, respectively, the dimension of our parameter is $[J]=[E] /[M]^{2},[\tilde{\lambda}]=[E] /[M],[h]=[E] /[M],\left[\rho_{F}\right]=[E]^{-1}, \Delta=[E]$ and $[T]=[E]$, where the $[\mathrm{E}]$ is the unit of energy and $[\mathrm{M}]$ is that of magnetization. Therefore, when we make a correspondence between the actual value of magnetization and our calculated $m$, and the actual transition temperature and our calculated $T_{N}$, we must multiply $m$ by the real value of $c$ for magnetization and $D$ for transition temperature. The value of $\rho_{F}$ are determined in the range where it is consistent to the specific heat jump observed by the experiment. For example, with setting the energy unit as $D=100 \mathrm{~K}$, the value $\rho_{F}=0.2$ gives the linear specific heat coefficient $\gamma \equiv 2 \pi^{2} k_{\mathrm{B}}^{2} \rho_{F} / 3$ as $110 \mathrm{~mJ} / \mathrm{K}^{2} \mathrm{~mol}$ which is about twince the decrease of $\gamma, 50 \mathrm{~mJ} / \mathrm{K}^{2} \mathrm{~mol}$, across the magnetic trantision.

The smallness of magnetization may be a manifestation of the fact that the actual value of $\Delta$ is larger than that frequently used so far. It is rather difficult task to determine the actual value of $c, D$ and $\Delta$ from experiments so that their frequently used value $\left(c=1.2 \mu_{\mathrm{B}}, D \sim 100 K\right.$ and $\Delta=120 K$ ) have ambiguity to some extent. In particular, if we estimate the Van Vleck contribution to the magnetic susceptibility, $2 c^{2} / \Delta$, with $\Delta=120 K$ and $c=1.2 \mu_{\mathrm{B}}$, we obtain twice as large value as the observed magnetic susceptibility $\left.\left(\sim 5 * 10^{-3} \mathrm{emu} / \mathrm{mol}\right)^{2}\right)$ at the zero temperature. Considering the fact that the observed value of the susceptibility further contains the contribution from the itinerant quasiparticles in general, we must take larger value of $\Delta$ than $\Delta=120 \mathrm{~K}$. In fact Santini et al have taken $\Delta=460 \mathrm{~K}$ and $c=1.6 \mu_{\mathrm{B}}$ to reproduce the obtained the susceptibility within the singlet ground crystal field scheme. So it is reasonable to take the large value of $\Delta$ as in our calculation. If we take $D=100 K$ and $c=1.6 \mu_{\mathrm{B}}$, our result of solid line in Fig. 1 implies that $T_{N}$ is about $7 \mathrm{~K}$ and $m$ is about $0.13 \mu_{\mathrm{B}}$. The magnitude of these values are different from those of observed values $\left(T_{N}=17.5 \mathrm{~K}\right.$ and $\left.m=0.04 \mu_{\mathrm{B}}\right)$ about numerical factors, but we obtain the same order as those of observed values of $T_{N}$ and $m$. The difference in the factor may be improved by changing the parameter value within the permitted range. For example, if we take much larger value for $\tilde{\lambda}$ and little value for $\rho_{F}$, we will obtain better result. Another improvement will be achieved if we consider the unit of energy $D$ to be larger than $(D=) 100 \mathrm{~K}$. $D$ is the 'band width' of itinerant fermion in the duality model and is further renormalized to the Fermi liquid fixed point. So the 
value of $D$ has ambiguity of order 1 . If we set $D=200 \mathrm{~K}$, for example, our result of $T_{N}$ reproduces the experimental value without making the other parameters out of the permitted range. Finally we should not forget the possibility of more suitable scheme of crystal field levels. We are based on the scheme of Santini $\mathrm{el}$ al's. However the difficulties of determination of crystal field levels remain the room for much larger (smaller) value of $\Delta(c)$ so that we might obtain better result for the $T_{N}$ and $m$.

Fig. 1. Temperature dependence of the ordered moment for various values of parameters. The parameters here are normalized by the unit $c$ for the magnetization and $D$ for the energy.

\subsection{Specific heat jump}

The specific heat jump $\Delta C$ at $T=T_{\mathrm{N}}$ is given in terms of the coefficients $\alpha$ and $\beta$ of the Ginzburg-Landau free energy as

$$
\Delta C=T_{\mathrm{N}} \frac{\alpha^{2}}{\beta} .
$$

Without the $m$-dependence of $\chi_{0}$, these coefficients are the same as those of BCS. The deviations from BCS are determined by expanding the equation of state, $(2.4 \mathrm{~b})$, with respect to $m$, and comparing the linear and cubic terms as follows:

$$
\alpha=\left(\tilde{\lambda}^{2} \rho_{\mathrm{F}}+\frac{1}{2 \sinh ^{2} \frac{\Delta^{2}}{2 T_{\mathrm{N}}}} \frac{\Delta}{8 c^{2} T_{\mathrm{N}}}\right) \frac{1}{T_{\mathrm{N}}}
$$




$$
\beta=\rho_{\mathrm{F}} \tilde{\lambda}^{4} \frac{7 \zeta(3)}{8 \pi^{2} T_{\mathrm{N}}^{2}}+\frac{(z J)^{2}}{2 \Delta} \operatorname{cth} \frac{\Delta}{2 T_{\mathrm{N}}}-\frac{(z J)^{2}}{2 T_{\mathrm{N}}} \frac{1}{\sinh ^{2} \frac{\Delta}{2 T_{\mathrm{N}}}}
$$

where the first terms are those for BCS. In the case of $\mathrm{URu}_{2} \mathrm{Si}_{2}$, it is expected that $\rho_{\mathrm{F}} \tilde{\lambda} \sim 1$, $\Delta \sim \tilde{\lambda} \gtrsim z J$, and $\Delta$ is several times larger than $T_{\mathrm{N}}$; so that the first terms in (3.10a) and (3.10b) are predominant reproducing the BCS value for $\Delta C$. Namely, we obtain a large specific heat jump as in the BCS or simple SDW systems and actually we reproduce the same order of experimental value of specific heat jump with the typical values of our parameters in section 3.3. The reason why Sikkema et al cannot reproduce the large specific heat jump13) is mainly due to their value of $\Delta$. They have taken the value $120 \mathrm{~K}$ for $\Delta$ by seeing the balance of other parameters in their simple Hamiltonian. We take rather large value of $\Delta$ and does not attach importance to keep the $\Delta=120 \mathrm{~K}$ because its value estimated from the experiment has ambiguity to some extent. Furthermore their specific heat jump are mainly due to the gap opening of the Fermi surface of the conduction electron which are not renormalized, but within our scheme the itinerant electron are renormalized and they bring large specific heat jump.

\subsection{Orbital order induced by antiferromagnetism}

The induced mean-field $h_{\text {eff }}$ determines the orbital state of f-electrons through the matrix element $c=<1\left|J_{z}\right| 0>$. Indeed, the effective Hamiltonian $H_{\mathrm{eff}}^{\text {loc }}$ for the local state is written as

$$
H_{\mathrm{eff}}^{\mathrm{loc}}=E_{0}|0><0|+\left(E_{0}+\Delta\right)|1><1|-h_{\mathrm{eff}} c(|0><1|+|1><0|),
$$

where $E_{0}$ is the energy of the crystal field state $\mid 0>$. The ground state $\mid \mathrm{g}>$ of $(3.11)$ is given by

$$
\mid \mathrm{g}>=\frac{1}{\sqrt{\left(h_{\mathrm{eff}} c\right)^{2}+\left(E_{\mathrm{g}}-E_{0}\right)^{2}}}\left(h_{\mathrm{eff}} c\left|0>+\left(E_{\mathrm{g}}-E_{0}\right)\right| 1>\right),
$$

where the ground state energy $E_{\mathrm{g}}$ is defined as

$$
E_{\mathrm{g}}=E_{0}+\frac{1}{2}\left[\Delta-\sqrt{\Delta^{2}+4\left(h_{\mathrm{eff}} c\right)^{2}}\right]
$$

This means that the local orbital state depends on the mean field $h_{\text {eff }}$. Therefore, the antiferromagnetic induced-moment, i.e., the mean field, gives rise to the orbital order of f-electrons because the induced moment is different between the sublattices. Note that the charge density corresponding to the state (3.12) depends on the sign of $h_{\text {eff }}$ through the cross term of $\mid 0>$ and $\mid 1>$. This may be a novel aspect of induced-moment antiferromagnetism which has not yet been recognized so far. This effect will give some physical response that suggests at first sight some 'hidden order' other than the antifferomagnetic order. But when induced moment is small as in this case, it is hard to detect the orbital order. Indeed ${ }^{101} \mathrm{Ru}$ NQR study has not detected the explicit signal of orbital order in $\mathrm{URu}_{2} \mathrm{Si}_{2}, 17$ 


\subsection{Effects of external field}

Here let us discuss the effect of external field on the ordered moment $m$ at zero temperature and the transition temperature $T_{\mathrm{N}}$. First, $m=\frac{1}{2}\left(S_{A}-S_{B}\right)$ is determined from eqs.(2.4) as follows:

$$
\begin{aligned}
\frac{1}{\chi_{0}(Q, S)}-z J-2 \tilde{\lambda}^{2} \rho_{\mathrm{F}} \Pi(Q, m ; h) & =0 \\
\left(\frac{1}{\chi_{0}(0, S)}+z J-2 \tilde{\lambda^{2}} \rho_{\mathrm{F}}\right) S_{0} & =h .
\end{aligned}
$$

where the polarization $\Pi$ is given as

$$
\begin{aligned}
\Pi(Q, m ; h) & =\log \frac{D+\sqrt{D^{2}+(\tilde{\lambda} m)^{2}}}{\tilde{h}+\sqrt{\tilde{h}^{2}-(\tilde{\lambda} m)^{2}}} \quad(\tilde{h}>\tilde{\lambda} m) \\
& =\log \frac{D+\sqrt{D^{2}+(\tilde{\lambda} m)^{2}}}{\tilde{\lambda} m} \quad(\tilde{\lambda} m>\tilde{h}) .
\end{aligned}
$$

Next, $T_{\mathrm{N}}$ under the external field is determined by the relations similar to eqs.(3.14):

$$
\begin{aligned}
\frac{1}{\chi_{0}(Q, S)}-z J-\frac{\tilde{\lambda}^{2} \rho_{\mathrm{F}}}{2} \int_{-D}^{D} \frac{\mathrm{d} \epsilon}{\epsilon}\left(\operatorname{th} \frac{\epsilon+\tilde{h}}{2 T_{N}}+\operatorname{th} \frac{\epsilon-\tilde{h}}{2 T_{N}}\right) & =0 \\
\frac{S_{0}}{\chi_{0}(0, S)}+z J S_{0}-2 \tilde{\lambda}^{2} \rho_{\mathrm{F}} S_{0} & =h .
\end{aligned}
$$

The critical field $h_{\mathrm{c}}$ given by (3.14), where $m \rightarrow 0$, and by (3.16), where $T_{N} \rightarrow 0$, coincides with each other of course. However, the $h$-dependence of $m$ and $T_{\mathrm{N}}$ at lower field $h<h_{\mathrm{c}}$ can exhibit rather different behaviors. Indeed, the numerical solutions of (3.14) and (3.16) are shown in Fig. 2 for the parameters seemingly relevant to $\mathrm{URu}_{2} \mathrm{Si}_{2}$. In Fig. 2 the numerical instability occurs for high field region $h \simeq h_{\mathrm{c}}$ in determining $m$ in (3.14), while we are concerned with the lower field region. We can see in Fig. 2 that the reduction rate of $m$ as increasing $h$ is much larger than that of $T_{\mathrm{N}}$ in the low field region. This result can be understood qualitatively as follows. The degree of nesting is rapidly destroyed by the external field at $T=0$, and so is $m$, while at $T_{\mathrm{N}}$ the degree of nesting has already been smeared to some extent by thermal effect from the beginning so that the effect of $h$ on $T_{\mathrm{N}}$ is more mild compared to the case of $m(T=0)$. The $h$-dependence shown in Fig. 2 is exactly observed in experiments of $\mathrm{URu}_{2} \mathrm{Si}_{2}$ ( 18 )

Experimental results are fit by the following phenomenological formulae which have different critical field for magnetic moment and transition temperature 618

$$
\begin{aligned}
m(h) & =m(0) \sqrt{1-\left(h / h_{\mathrm{c}_{1}}\right)^{3 / 2}}, \\
T_{N}(h) & =T_{N}(0)\left[1-\left(\frac{h}{h_{\mathrm{c}_{2}}}\right)^{2}\right],
\end{aligned}
$$

For comparison with our theoretical curves, we show these phenomenological forms by dotted lines with $h_{\mathrm{c}_{1}}=1.0$ and $h_{\mathrm{c}_{1}}=2.15$. One can see good agreement between the phenomenological formulae and our theoretical results in the low field region. These phenomenological formulae are derived 
Fig. 2. The ordered moment $\mathrm{m}$ and the transition temperature $T_{N}$ vs external magnetic field $\mathrm{h}$.

As in Fig. 1 we take $c$ as the unit of magnetization and $D$ that of energy. The solid lines are our theoretical results for $m(h)$ and $T_{N}(h)$, while the dotted and dashed ones are phenomenological formulae $(3.17 \mathrm{a}, \mathrm{b})$ with $h_{\mathrm{c}_{1}}=1.0$ and $h_{\mathrm{c}_{2}}=2.15$. The dashed ones represent the curves for $m(h) / m(0)>0.7$ and $T_{N}(h) / T_{N}(0)>0.6$ as experiment by Mentink et al. 6 We can see good agreement between our results and the phenomenological curves for low field region.

below the field at $m(h) / m(0) \sim 0.7$ and $T_{N}(h) / T_{N}(0) \sim 0.6$, 618 so our results can be regarded to reproduce the experimental fact that the magnetic moment at $T=0$ and the transition temperature appears to have deferent critical fields. Mentink et al have claimed the existence of some hidden order parameters from these different dependence on magnetic field, we have no need for such an exotic order in our scheme. These unconventional features cannot be obtained only from the nesting property, but are the results of the interplay between the induced-moment mechanism of "localized spin" and the nesting property of "itinerant fermion" which are well described by the framework of duality model in a unified way.

\section{$\S 4$. Conclusion}

We have shown within the mean-field approximation on the basis of itinerant-localized duality model that the small ordered moment and large specific heat jump observed in $\mathrm{URu}_{2} \mathrm{Si}_{2}$ can be explained by the nesting property of Fermi surface with considering the singlet-singlet crystal field scheme. This model also explains the apparently different field dependence of the transition

temperature and the magnetic moment observed by the neutron scattering experiments. These "anomalous" magnetic feature of $\mathrm{URu}_{2} \mathrm{Si}_{2}$ does not necessarily need the hidden exotic order param- 
eter 19. The nesting feature of Fermi surface of $\mathrm{URu}_{2} \mathrm{Si}_{2}$ is supported by the band calculation.20) In addition the induced antiferromagnetism naturally invokes the orbital order which causes the effects that cannot be understood by simple SDW antiferromagnetism.

The realization of observed small moment stands in a delicate balance of physical parameters to some extent. We believe that the values of parameters we take in this papers are not so unrealistic and the situation of real system actually stands in such a balance of physical quantities. Moreover if we go beyond the mean field level and take into account quantum fluctuations, we will be able to obtain improved results in quantitative level.

In this paper we have not taken into account explicitly the nature of the $\mathrm{f}^{2}$-configuration. Watanabe and Kuramoto recently pointed out a possibility of new kind of metal-insulator transition between the localized state $\mathrm{f}^{2}$-configuration with crystalline electric field singlet and the itinerant

state with the Kondo screening.21 So there may exist the situations of $\mathrm{URu}_{2} \mathrm{Si}_{2}$ in which we have to consider the nature of $\mathrm{f}^{2}$-configuration explicitly as they did.

There remains some other important problems for $\mathrm{URu}_{2} \mathrm{Si}_{2}$; for example, the relation between the magnetism and the superconductivity, the properties of quasi-particle in the $\mathrm{f}^{2}$-configuration with singlet ground state of crystal field,22 and so on. These are left for future investigations.

\section{Acknowledgement}

We are deeply indebted to Professor Y. Kuramoto for valuable discussions and critical reading of the manuscripts. One of the author (K.M.) has benefited from simplified model calculations at very preliminary stage by Kyoko Asano and from conversations with N. van Dijk. This work is supported by the Grant-in-Aid for Scientific Research (07640477) and the Grand-in-Aid for Scientific Research on Priority Areas "Physics of Strongly Correlated Conductors" (06244104) from the Ministry of Education, Science, Sports and Culture.

[1] W. Schlabitz, J. Baumann, B. Pollit, U. Rauchschwalbe, H. M. Mayer, U. Ahlheim and C. D. Bredl: Z. Phys. B 62 (1986) 171.

[2] B. Fåk, C. Vettier, J. Flouquet, F. Bourdarot, S. Raymond, A. Vernière, P. Lejay, Ph. Boutrouille, N. R. Bernhoeft, S. T. Bramwell, R. A. Fisher and N. E. Phillips: J. Magn. Magn. Mat. 154 (1996) 339.

[3] C. Broholm, J. K. Kjems, W. J. L. Buyers, P. Matthews, T. T. M. Palstra, A. A. Menovsky, and J. A. Mydosh: Phys. Rev. Lett 58 (1987) 1467.

[4] T. T. M. Palstra, A. A. Menovsky, J. van den Berg, A. J. Dirkmaat, P. H. Kes, G. J. Nieuwenhuys and J. A. Mydosh: Phys. Rev. Lett. 55 (1985) 2727.

[5] M. B. Maple, J. W. Chen, Y. Dalichaouch, T. Kohara, C. Rossel, M. S. Torikachvili, M. W. McElfesh and D. J. Thompson: Phys. Rev. Lett. 56 (1986) 185.

[6] S. A. M. Mentink, T. E. Mason, S. Süllow, G. J. Nieuwenhuys, A. A. Menovsky, J. A. Mydosh and J. A. A. J. Peerenboom: Phys. Rev. B 53 (1996) R6014.

[7] P. Santini and G. Amoretti: Phys. Rev. Lett. 73, (1994) 1027

[8] G. J. Niewenhuys: Phys. Rev. B 35 (1987) 5263. 
[9] R. J. Radwański: J. Magn. Magn. Mater. 103 (1992) L1.

[10] C. Broholm, H. Lin, P. T. Matthews, T. E. Mason, W. J. L. Buyers, M. F. Collins, A. A. Menovsky, J. A. Mydosh and J. K. Kjems: Phys. Rev. B 43 (1991) 12809.

[11] Y.-L. Wang and B. R. Cooper: Phys. Rev. 185 (1969) 696.

[12] B. Grover: Phys. Rev. 140 (1965) A1944.

[13] A. E. Sikkema, W. J. L. Buyers, I. Affleck and J. Gan: Phys. Rev. B 54 (1996) 9322

[14] Y. Kuramoto and K. Miyake: J. Phys. Soc. Jpn. 59 (1990) 2831

[15] Y. Kuramoto and K. Miyake: Prog. Theor. Phys. 108 (1992) 199.

[16] Y. Okuno, O. Narikiyo and K. Miyake: J. Phys. Soc. Jpn. 66 (1997) 2389.

[17] K. Matsuda, Y. Kohori and T Kohara: Physica, B 230-232 (1997) 351.

[18] J.-G. Park, K. A. McEwen, S. de Brion, G. Chouteau, H. Amitsuka and T. Sakakibara: J. Phys. C 9 (1997) 3065 .

[19] V. Barzykin and L. P. Gor'kov: Phys. Rev. Lett. 74 (1995) 4301.

[20] G. J. Ronzing, P. E. Mijnareds and D. D. Koelling: Phys. Rev. B. 43 (1991) 9515.

[21] S. Watanabe and Y. Kuramoto: preprint cond-mat/9706134

[22] H. Ikeda and K. Miyake: J. Phys. Soc. Jpn. 66 (1997) 3714. 


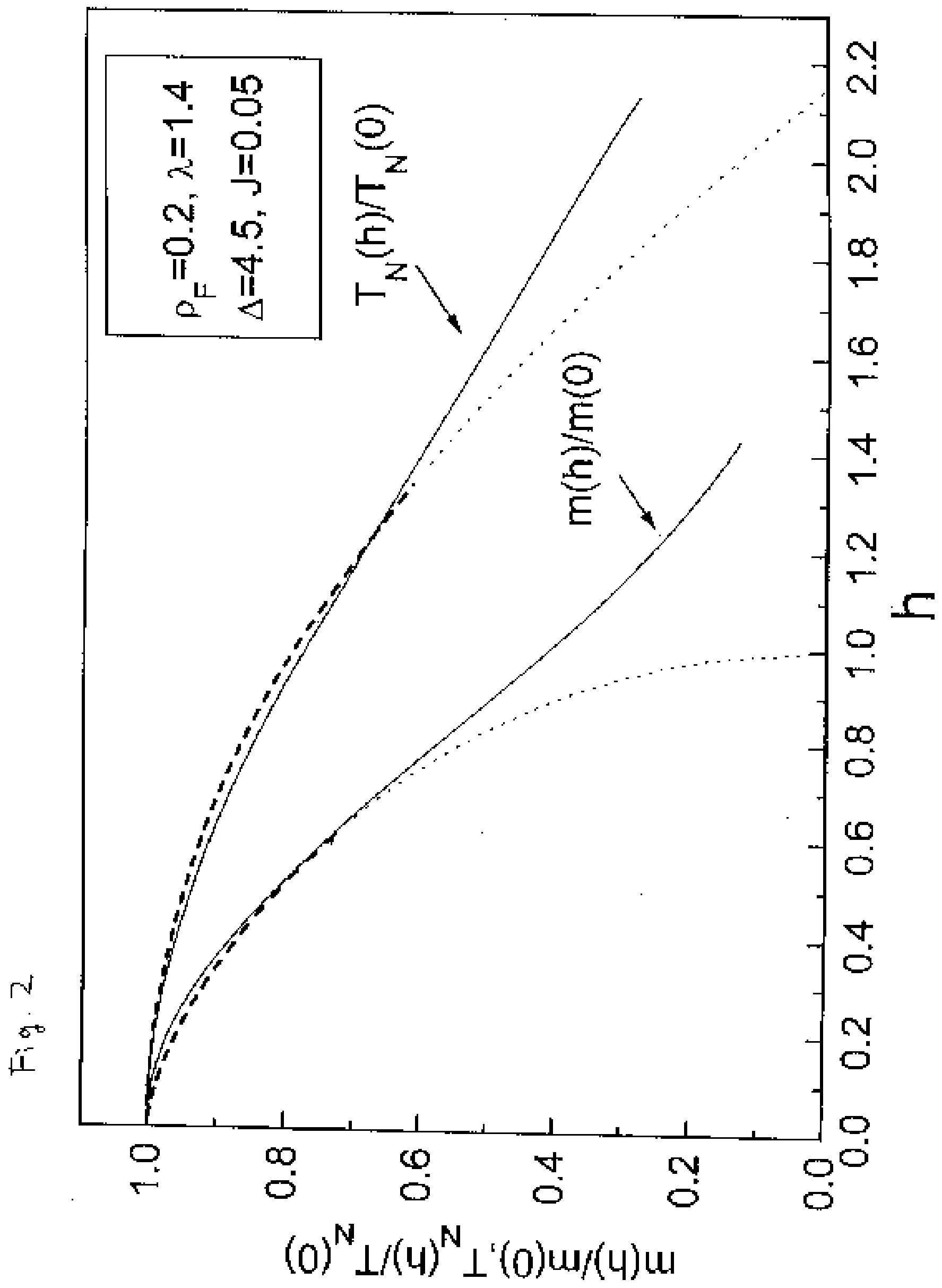

